\title{
Capacidade funcional e envolvimento social em idosos: há relação?
}

\author{
Francine Náthalie F. R. Pinto*, Dayane Capra de Oliveira*
}

\section{Resumo}

Um dos maiores desafios da atualidade é gerenciar o processo de envelhecimento da população e, com isso, o consequente aumento das doenças crônico-degenerativas. O desenvolvimento de incapacidades pode estar associado ao aumento na manifestação dessas doenças e ao despreparo do sistema de saúde, podendo levar os idosos a situações de total dependência de outrem e ao isolamento social, desencadeando um processo de declínio funcional. O objetivo do presente estudo foi realizar uma revisão da literatura que abordasse a temática: o envolvimento social de idosos e sua capacidade funcional. Para isso, realizou-se um levantamento bibliográfico nas seguintes bases de dados: LILACS, Redalyc, BVS e SciELO. Foram encontrados quatro artigos que atenderam ao critério de inclusão. Os estudos indicaram que o envolvimento social de idosos e a presença de um cônjuge são fatores protetivos, ou seja, contribuem para a manutenção da sua capacidade funcional. Concluiu-se, então, que a independência funcional é um fator preditivo para o envelhecimento bem sucedido. Dessa forma, entende-se que as re- lações sociais devem ser desenvolvidas ao longo da vida, para que, na velhice, elas se mantenham, uma vez que uma vida social ativa pode influenciar positivamente na manutenção da capacidade funcional dos idosos. As atividades de trabalho e de lazer também devem ser valorizadas.

Palavras-chave: Interação. Relacionamento. Idosos. Envelhecimento. Qualidade de vida.

\section{Introdução}

Devido a melhores condições médico-sanitárias, ao planejamento familiar e, consequentemente, à redução do número de jovens, e ao aumento da população idosa, o Brasil está envelhecendo, e com isso deve passar por profundas transformações socioeconômicas (IBGE, 2013). Estima-se que no Brasil, nos próximos vinte anos, a população de idosos aumentará exponencialmente, e que, em 2060, a população com essa faixa etária deve

* Mestre em Psicologia pela UFSCar, doutoranda do Programa de Pós-Graduação em Psicologia, Universidade Federal de São Carlos, São Carlos-SP. Departamento de Psicologia. Rodovia Washington Luís, km 235, CEP: 13565-905 - São Carlos-SP, Brasil. E-mail: francinenaty@yahoo.com.br

** Fisioterapeuta, especialista em Fisioterapia Geriátrica pela Universidade Federal de São Carlos, São Carlos-SP. E-mail: dayacapra@hotmail.com

$\rightarrow$ http://dx.doi.org/10.5335/rbceh.v12i1.4687

Recebido em: 23/03/2015. Aceito em: 18/05/2015 
passar de 58,4 milhões. A expectativa média de vida do brasileiro deve aumentar de 75 para 81 anos (IBGE, 2013).

Um dos maiores desafios da saúde pública atual é gerenciar o acentuado processo de envelhecimento populacional e o aumento das doenças crônico-degenerativas. A presença de vários tipos de doenças crônico-degenerativas pode estar associada ao crescimento na porcentagem de idosos incapazes e dependentes, uma vez que essas doenças podem levar à permanência restrita no domicílio e ao isolamento social, desencadeando, por sua vez, um processo de declínio da capacidade funcional desses idosos (GRATÃO et al., 2013). No Brasil, aproximadamente $40 \%$ dos idosos com idade acima de 65 anos dependem de outrem para algum tipo de tarefa, e esta ajuda é predominantemente dada por algum membro da família (UESUGUI; FAGUNDES; PINHO, 2011).

A capacidade funcional $(\mathrm{CF})$ pode ser definida como a habilidade do idoso em desempenhar tarefas de forma independente no seu cotidiano (FARIAS; BUCHALLA, 2005; FIDLER; PERES, 2008). Para avaliar a CF de um idoso, deve-se avaliar também o desempenho funcional nas atividades básicas de vida diária (ABVD) e nas atividades instrumentais da vida diária (AIVD) (FREITAS et al., 2012). As ABVD envolvem atividades de autocuidado, tais como: alimentar-se, vestir-se, banhar-se, mobilizar-se e deambular; e as AIVD se referem às atividades que proporcionam independência no lar e em demais atividades do indivíduo, como manipular medicamentos, administrar as próprias finanças, realizar compras e utilizar os meios de transporte (FARIAS; BUCHALLA, 2005; FREITAS et al., 2012). A preservação da capacidade de realizar essas atividades, seja nas funções dentro de casa ou no convívio em sociedade, é essencial para a independência do idoso em sua comunidade (FARIAS; BUCHALLA, 2005; TÚBERO et al., 2013). Segundo Gratão et al. (2013, p. 141),

[...] a independência do idoso está relacionada diretamente à sua capacidade em desenvolver as atividades da vida diária sem auxílio, à autonomia e à liberdade em decidir por sua própria vontade, gerenciando sua vida.

Já a incapacidade refere-se a dificuldades em realizar atividades cotidianas (ALVES; LEITE; MACHADO, 2010).

Conforme Teixeira e Neri (2008), um envelhecimento bem sucedido é acompanhado de qualidade de vida e de sentimento de bem-estar, e deve ser desenvolvido ao longo da trajetória de vida. A interação multidimensional entre a saúde física, a saúde mental, a independência na vida diária, a integração social, o suporte familiar e a independência econômica passa a ser resultante de um processo de envelhecimento saudável (CUPERTINO; ROSA; RIBEIRO, 2007; TEIXEIRA; NERI, 2008). Um idoso que apresente uma boa capacidade funcional e ausência de problemas de outra ordem (como incidência de violência doméstica no lar, depressão, entre outros) desfrutaria de bem-estar na velhice (CUPERTINO; ROSA; RIBEIRO, 2007; TEIXEIRA; NERI, 2008). De outro modo, quando o idoso perde sua capacidade funcional, ele passa a ter uma maior probabilidade 
de apresentar outros problemas além da dependência em si, como, por exemplo, maior probabilidade de ter depressão, ansiedade, sentimentos de solidão, conflitos familiares, entre outros (GRATÃO et al., 2013; PINTO; BARHAM; ALBUQUERQUE, 2013). Uma forma de prevenir as vulnerabilidades físicas e sociais é por meio do envolvimento dos idosos em atividades sociais, ou ofertando a eles algum tipo de suporte social (BRITO; PAVARINI, 2012; ROSA; CUPERTINO; NERI, 2009).

De acordo com Neri e Vieira (2013), o envolvimento social de idosos é parte fundamental para uma velhice bem sucedida, e esta está relacionada a atividades avançadas da vida diária, nas quais os idosos são capazes de ir à igreja, fazer viagens curtas, e ir a reuniões sociais. Em conjunto com o envolvimento social, na área de gerontologia, fala-se também da importância do apoio social dado pela família aos idosos (SERBIM; GONÇALVES; PASKULIN, 2013), pois quando recebem algum tipo de apoio, eles se mantêm ativos e se relacionando socialmente. $\mathrm{O}$ apoio social é uma troca entre as pessoas, no sentido de promover o bem-estar físico e psicológico (NERI; VIEIRA, 2013). Esse apoio pode se dar por meio de ajuda nas tarefas do dia a dia, como afeto e companhia ao idoso, fazendo com que ele se sinta amado e valorizado. $\mathrm{O}$ apoio social da família ajuda os idosos no processo de enfrentamento e na recuperação de enfermidades, estresse e outras experiências difíceis da vida (SERBIM; GONÇALVES; PASKULIN, 2013).
No Brasil, a família é responsável por assistir seus familiares idosos (BRASIL, 2004; PINTO; BARHAM; ALBUQUERQUE, 2013). Há dados que indicam que quando os idosos se sentem mais sozinhos, eles têm maiores chances de desenvolver incapacidades funcionais e, nesse sentido, necessitar da ajuda de outrem com o passar do tempo (GRATÃO et al., 2013). Diante disso, torna-se essencial estudar a influência que o envolvimento em atividades sociais tem na manutenção da capacidade funcional dos idosos. Além disso, é necessário considerar o efeito do envolvimento social na saúde dos idosos e seus efeitos no bem-estar psicológico e na satisfação com a vida, uma vez que há estudos que mostram o caráter protetivo de se manter ativo e envolvido com atividades sociais e de lazer para essa população (BRITO; PAVARINI, 2012; FERREIRA; BARHAM, 2011; NERI; VIEIRA, 2013; TEIXEIRA; NERI, 2008).

Dessa forma, o envolvimento social de idosos se mostra altamente relevante, já que pode ajudar na prevenção de incapacidades funcionais, mantendo os sujeitos mais ativos e, consequentemente, dando maiores chances de um envelhecimento com qualidade de vida. Assim, o presente estudo teve como objetivo realizar uma revisão da literatura que abordasse a temática: o envolvimento social de idosos e sua capacidade funcional.

\section{Método}

Para a elaboração deste trabalho, realizou-se uma pesquisa bibliográfica nas seguintes bases de dados: LILACS, 
Redalyc, Biblioteca Virtual de Saúde (BVS) e SciELO, sem limitação de ano de publicação, de forma que foram consultados todos até janeiro de 2015. Foram utilizados os seguintes descritores: capacidade funcional, envolvimento social, relacionamento social, interação social, relacionamento interpessoal, idosos, idoso, envelhecimento. Esses descritores foram escolhidos para que fosse possível relacionar a variável envolvimento social com a capacidade funcional.

Foram incluídos artigos que tratassem do envolvimento social de idosos e os relacionasse com capacidade funcional, e estivessem escritos na língua portuguesa. Foram excluídos artigos que relacionavam capacidade funcional somente com dados sociodemográficos ou outras variáveis.

\section{Resultados}

Inicialmente, após a inserção dos descritores, foram encontrados 34 artigos. Após a leitura dos resumos, quatro artigos se encaixaram no perfil delimitado para este estudo. Dentre os 34 artigos, 30 foram excluídos por relacionarem capacidade funcional somente com dados sociodemográficos ou por relacionarem capacidade funcional com apoio social, e não com o envolvimento social em si. Os artigos foram analisados nos seguintes aspectos: objetivos, método e evidências obtidas.

Os quatro artigos selecionados, então, são nacionais e foram publicados em revistas brasileiras, conforme descrito na Tabela 1.

Tabela 1 - Estudos sobre capacidade funcional e envolvimento social de idosos, publicados entre 2003 e 2013

\begin{tabular}{l|l|l}
\hline \multicolumn{1}{c|}{ Artigo } & \multicolumn{1}{c|}{ Autores } & \multicolumn{1}{c}{ Periódico } \\
\hline $\begin{array}{l}\text { Doenças crônicas, capacidade funcional, } \\
\text { envolvimento social e satisfação em ido- } \\
\text { sos comunitários: Estudo Fibra }\end{array}$ & Pinto e Neri (2013) & Ciência e Saúde Coletiva \\
$\begin{array}{l}\text { Trabalho, suporte social e lazer protegem } \\
\text { idosos da perda funcional: Estudo Epidoso }\end{array}$ & $\begin{array}{l}\text { D'Orsi, Xavier e Ramos } \\
(2011)\end{array}$ & $\begin{array}{l}\text { Revista de Saúde Pública } \\
\begin{array}{l}\text { Fatores determinantes da capacidade } \\
\text { funcional em idosos longevos }\end{array}\end{array}$ \\
$\begin{array}{l}\text { Fatores determinantes da capacidade } \\
\text { funcional entre idosos }\end{array}$ & Rosa et al. (2003) & $\begin{array}{l}\text { Revista de Saúde Pública } \\
\text { sioterapia }\end{array}$ \\
\hline
\end{tabular}

\section{Objetivos dos estudos}

Os estudos apresentaram objetivos com forte relação quanto à temática capacidade funcional e envolvimento social, relacionando, dessa forma, o en- volvimento social de idosos com a sua capacidade funcional. $\mathrm{O}$ artigo de Pinto e Neri (2013) teve como objetivo descrever as variáveis: envolvimento social, capacidade funcional, presença de doenças, memória, idade, renda, e em seguida 
realizar correlações entre envolvimento social e capacidade funcional em idosos independentes. $\mathrm{O}$ artigo de D'Orsi, Xavier e Ramos (2011) teve como objetivo identificar fatores de risco para perda da capacidade funcional de idosos. Já o artigo de Nogueira et al. (2010) teve como objetivo investigar a influência de fatores socioeconômicos, demográficos, biológicos e das relações sociais sobre a capacidade funcional de idosos longevos. E, por último, o objetivo de Rosa et al. (2003) foi investigar a influência de fatores socioeconômicos, demográficos e de fatores ligados às atividades sociais sobre a capacidade funcional dos idosos. Percebe-se que os estudos de Nogueira et al. (2010) e de Rosa et al. (2003) têm objetivos bem similares, e que todos buscam verificar, de alguma forma, uma possível relação entre capacidade funcional e envolvimento social.

\section{Descrição metodológica dos estudos}

Os participantes dos estudos analisados eram em sua totalidade idosos. O número de participantes variou de 129 (NOGUEIRA et al., 2010) a 2472 (PINTO; NERI, 2013) idosos. Em todos os estudos, a maioria dos participantes era do sexo feminino.

Pinto e Neri (2013) avaliaram 2472 idosos sem déficit cognitivo quanto à presença de doenças crônicas, envolvimento social, memória e dados sociodemográficos, por meio de autorrelato. A capacidade funcional foi avaliada por meio de um dinamômetro hidráulico que mediu a força de preensão palmar e a velocidade da marcha.
Já D’Orsi, Xavier e Ramos (2011) acompanharam um único grupo de pacientes por dez anos, em quatro encontros domiciliares para cada participante, realizado entre 1991 e 2001, totalizando 1667 idosos entrevistados inicialmente. No entanto, desses, somente 326 permaneceram até o final, devido a óbitos, mudanças de endereço ou recusa. A primeira entrevista foi realizada em 1991 e 1992; a segunda em 1994 e 1995; a terceira em 1998 e 1999; e a quarta em 2000 e 2001. As entrevistas utilizaram o Questionário Brasileiro de Avaliação Funcional Multidimensional (BomfaqRAMOS et al., 1998). Nesses encontros, foram coletadas as informações sobre características socioeconômicas, demográficas, suporte informal, grau de independência nas AVD, doenças crônicas, saúde mental, cognição e autopercepção de saúde dos idosos.

No entanto, os autores não explicaram como foi a coleta de dados sobre o suporte informal dos idosos, só informaram que este tipo de suporte era provido por pessoas não pertencentes a nenhum programa do governo ou instituição especializada. O objetivo desses quatro encontros foi comparar a capacidade funcional desses idosos com o passar dos anos. Foram selecionados os idosos considerados como independentes na primeira entrevista ou com dependência leve (comprometimento de 1 a 2 AVD/AVDI). Definiu-se como perda funcional a dependência em sete ou mais AVDs/AVDIs. Aqueles que apresentaram perda funcional na segunda ou na terceira entrevista foram identificados e comparados aos que não apresentaram nenhuma perda até então. 
Nogueira et al. (2010) estudaram 129 idosos longevos não institucionalizados, realizaram entrevistas e medições antropométricas de peso e altura nas residências de cada um. A partir das medidas antropométricas, obtiveram o Índice de Massa Corporal (IMC) para o diagnóstico nutricional, e por meio de questionários, analisaram as características: socioeconômicas e demográficas (gênero, faixa etária, escolaridade, estado civil, renda, história e tempo de residência na zona rural); biológicas e de saúde (uso de serviços de saúde e de medicamentos, visão e audição autorreferidas, ocorrência de quedas nos últimos três meses, morbidades autorreferidas e a polifarmácia - uso simultâneo de cinco ou mais medicamentos); relações sociais (visitar amigos e/ou parentes pelo menos uma vez por semana, participar de obras sociais, participar de alguma comunidade religiosa, participar de eventos sociais) e autoavaliação da saúde (saúde autorreferida e saúde em comparação com seus pares).

Rosa et al. (2003) avaliaram 964 idosos quanto à sua capacidade funcional. Esses idosos foram separados em dois grupos: (1) ausência de dependência $(\mathrm{N}=729)$ versus dependência leve $(\mathrm{N}=398)$ e (2) ausência de dependência (os mesmos 729) versus presença de dependência moderada/grave $(\mathrm{N}=235)$. Nesse estudo, foram apresentados somente os resultados referentes à dependência moderada ou grave devido à semelhança dos resultados obtidos nos dois grupos. Investigou-se a possível associação entre dependência funcional e as variáveis socioeconômicas (renda mensal, escolaridade e situação ocupacional), demográficas (sexo, idade, estado civil, local da residência, composição familiar e situação de propriedade do domicílio em que vive), saúde (hospitalização nos últimos seis meses, visão, audição, patologias, insônia, quedas e saúde mental), relações sociais (fazer atividades físicas, ir ao cinema/teatro/ concertos, visitar amigos e parentes, receber visitas, participar de obras religiosas, gosta/gostaria de trabalhar) e autopercepção de saúde (estado de saúde comparado com a saúde de seus pares). Dentre os estudos apresentados, o de D’Orsi, Xavier e Ramos (2011) foi o único longitudinal, os outros foram estudos correlacionais.

\section{Evidências apontadas nos estudos}

No estudo de Pinto e Neri (2013), constatou-se que as mulheres apresentavam mais doenças e tiveram pior desempenho funcional do que os homens, apesar de terem um maior envolvimento social. Além disso, a renda estava relacionada com o desempenho funcional, ou seja, os mais pobres apresentaram pior desempenho funcional. No que diz respeito às correlações, houve correlação positiva entre a capacidade funcional e o envolvimento social.

Já D’Orsi, Xavier e Ramos (2011) concluíram que há um maior risco de perda funcional para os idosos acima de 75 anos, com pior autopercepção de saúde e que tenham algum tipo de comprometimento cognitivo. Desenvolver atividades manuais, assistir TV e participar de jogos de salão foram encontrados como fatores de proteção. 
A atividade sexual também foi fator de proteção, assim como a manutenção do trabalho remunerado. Idosos que relataram relacionamento mensal com amigos e ter um amigo confidente apresentaram menor risco de perda funcional. Fatores de risco para perda da capacidade funcional foram hipertensão arterial, asma e diabetes.

Nogueira et al. (2010) encontraram evidências de que as chances de um idoso com mais de 85 anos apresentar dependência funcional é três vezes maior do que a de um idoso mais novo (por exemplo, um idoso com idade entre $60 \mathrm{e}$ 70 anos). Além disso, a chance de uma idosa apresentar pior capacidade funcional é seis vezes maior se comparada a de um idoso do sexo masculino. Fazer uso de vários medicamentos simultaneamente, considerar sua saúde pior que a de seus pares e não realizar atividades sociais semanais também foram fatores relacionados à incapacidade funcional.

E, por último, no estudo de Rosa et al. (2003), foi encontrado que as variáveis que se relacionaram com perda da capacidade funcional pelos idosos foram: analfabetismo, ser aposentado, ser pensionista, ser dona de casa, não ser proprietário da moradia, ter mais de 65 anos, ter composição familiar multigeracional, ter sido internado nos últimos seis meses, ser "caso" no rastreamento de saúde mental, não visitar amigos ou parentes, ter problemas de visão, ter histórico de AVC, e ter avaliação pessimista da saúde ao se comparar com seus pares.

De maneira geral, pode-se observar que mulheres apresentam pior capacidade funcional do que homens, e que uma maior renda está relacionada com uma maior capacidade funcional. Além disso, idosos que apresentam algum tipo de doença também tem maiores chances de terem piora em sua capacidade funcional. No entanto, confirmando a hipótese deste estudo, idosos que tem um envolvimento social maior (realizar atividades de lazer com amigos, se relacionar sexualmente, participar de encontros familiares, entre outros) tem sua capacidade funcional mais preservada ao longo de sua vida do que idosos em situações similares, mas que não tem um envolvimento social ativo.

\section{Discussão}

Visto que os estudos tiveram, de forma geral, objetivos semelhantes, suas conclusões também apresentaram resultados semelhantes. Todos eles relacionaram uma maior capacidade funcional a um maior envolvimento social no dia a dia dos idosos estudados. Percebe-se, então, a importância de os idosos manterem-se socialmente ativos, uma vez que se verificou que as atividades sociais podem atuar como fator de proteção da capacidade funcional de idosos, e são fundamentais para uma melhor qualidade de vida ao envelhecer (PINTO, NERI, 2013).

A presente revisão objetivou analisar e discutir os benefícios proporcionados pelo envolvimento social de idosos como forma de prevenção à diminuição de sua capacidade funcional. Diante disso, destaca-se que as boas relações sociais e familiares são fatores importantes para a manutenção da qualidade de vida de 
idosos, uma vez que o fato de o idoso ter boas relações pode contribuir para um maior envolvimento social do idoso com sua própria família e, principalmente, com pessoas de outras faixas etárias (BRITO; PAVARINI, 2012; PINTO, 2012; PINTO; BARHAM, 2014).

Um dos determinantes para uma boa qualidade de vida é a autonomia por parte dos idosos, sendo um dos pressupostos básicos para a tomada de decisão. Os idosos que tem autonomia têm, também, liberdade para gerenciar a própria vida, que é considerada condição primordial para o processo de envelhecimento prazeroso e bem sucedido (PINTO; NERI, 2013; SANTOS et al., 2012). A falta de autonomia, então, leva o idoso a ter uma diminuição na liberdade de tomar decisões, e pode estar relacionada à necessidade de maior cuidado dos familiares, considerando as possíveis complicações de alguma doença (GRATÃO et al., 2013).

O estudo de D’Orsi, Xavier e Ramos (2011) demonstrou que o aumento da idade é um fator de risco para a perda funcional, mas que a presença de trabalho remunerado e de atividades sociais podem ajudar a prevenir ou retardar a perda funcional por parte dessa população.

Outros estudos relataram associação entre o aumento da idade e a perda funcional, expondo idosos autônomos à síndrome de fragilidade. Diante disso, é fundamental que quanto mais avançar o envelhecimento, mais se trabalhe em função de manter as atividades, sejam elas sociais ou laborais, sempre considerando suas limitações (ALVES; LEITE; MACHADO, 2010; PINTO; NERI, 2013).
É importante reafirmar que os laços sociais (familiares e não familiares) agem como fator de proteção, e é possível afirmar que o suporte social decorrente do relacionamento mensal com os amigos protege da perda funcional, mostrando, assim, a importância das relações sociais afetivas, especialmente as de amizade para o envelhecimento ativo saudável (CUPERTINO; ROSA; RIBEIRO, 2007; FERREIRA; BARHAM, 2011; SIMÕES, 2010).

Considerando o estudo de Nogueira et al. (2010), observou-se que $71 \%$ dos longevos estudados apresentaram capacidade funcional boa ou muito boa, o que significa um alto índice de independência nessa população, sobretudo porque se refere a indivíduos com idade avançada, no entanto, o fato de eles terem chegado a uma idade avançada pode estar associado a ter uma vida que preservasse suas capacidades. Outro dado interessante foi a verificação de que há presença de uma menor capacidade funcional nas mulheres quando comparadas aos seus pares do sexo masculino. O maior risco de incapacidade entre as idosas pode ser atribuído à sua maior sobrevida e também à ligeira incapacidade física apresentada por elas na vida adulta, o que leva ao maior risco de desenvolverem algum grau de incapacidade funcional.

A vida social do idoso parece interferir de forma positiva na memória, além de melhorar a CF (NOGUEIRA et al., 2010). Além disso, idosos mais integrados na comunidade apresentam perda de memória mais lenta do que aqueles sem vida social ativa, além de terem menor chance de apresentar depressão (FERREIRA; BARHAM, 2011; NOGUEIRA et al., 2010). 
Observa-se também que altos índices de atividades sociais, tais como participar de eventos comunitários, fazer ou receber visitas e frequentar eventos religiosos, também estão associados a uma melhor capacidade funcional por parte dos idosos (PINTO; NERI, 2013; TORRES, 2013).

A presença de depressão é, hoje, considerada um importante preditor de incapacidade, com desencadeamento e agravamento do declínio funcional. Os fatores externos, como falta de rendimentos, de suporte social ou de recursos sociais, afetam a capacidade da pessoa em adaptar-se a novos contextos, desencadeando quadros depressivos que podem levar a uma repercussão negativa sobre a qualidade de vida dos idosos, com risco de maior mortalidade e suicídio (FERREIRA; BARHAM, 2011; SANTOS et al., 2012).

$\mathrm{O}$ gênero feminino foi uma variável interessante, pois acredita-se que, em função de as mulheres viverem mais do que os homens, elas experimentam um maior número de doenças crônicas e comorbidades, resultando em limitações funcionais e incapacidades durante a velhice.

O estado civil e a dependência funcional demonstraram forte associação, pois idosos casados ou que mantêm relação conjugal estável apresentaram menor probabilidade de limitação funcional (FREITAS et al., 2012; SERBIM; GONÇALVES; PASKULIN, et al., 2013), fato que também confirma a hipótese de que idosos com algum tipo de envolvimento social se mantêm mais funcionalmente capazes, uma vez que pelo menos com o parceiro há interação. Segundo Santos et al. (2012), idosos casados ou com companheiros têm uma melhor qualidade de vida e relacionamentos sociais mais ricos, uma vez que a família se mostra importante em todas as fases da vida, pois fortalece as relações e influencia a segurança emocional dos idosos.

Dessa forma, é de extrema importância que idosos que não têm um companheiro ou que ficaram viúvos recentemente tentem ampliar as suas redes sociais, pois as trocas de experiências com outras pessoas, pode influenciar diretamente sua qualidade de vida. Essas trocas podem ser atividades rotineiras, como fazer ou receber visitas e frequentar a igreja (PINTO, NERI, 2013; ROSA et al., 2003).

Além disso, ações que visem prevenir a perda da capacidade funcional são importantes, como a promoção e a formação de grupos de idosos ou o estímulo para uma vida social e saudável por meio da realização de atividades recreativas, físicas e culturais. Conforme Brito e Pavarini (2012), a relação entre a saúde dos idosos e o envolvimento social mostra que relações sociais satisfatórias podem promover melhores condições de saúde, no entanto, os mecanismos pelos quais esses efeitos são exercidos ainda não são totalmente conhecidos.

As relações sociais, além de serem um fator protetivo na manutenção da capacidade funcional dos idosos, auxiliam na manutenção das funções cognitivas (ALVES; LEITE; MACHADO, 2010; BRITO; PAVARINI, 2012). Por isso, deve-se estimular familiares e pessoas envolvidas na vida da população idosa a dar apoio e afeto, seja por meio de confiança, empatia, carinho, amor, escuta, seja pelo apoio às atividades diárias efetivamente, como, por exemplo, levar ao médico 
para consultas de rotina ou, até mesmo, acompanhar ao supermercado. As ações simples do dia a dia podem fazer com que o idoso sinta que está sendo acolhido e que tem suas necessidades supridas de forma integral, sejam elas instrumentais ou psicológicas.

De acordo com Torres (2013), as relações de proximidades emocionais devem ser incentivadas para que os idosos mantenham um sentimento de que pertencem ao meio no qual estão inseridos, para que, por meio desses sentimentos, ele tenha benefícios à saúde, fisiológica ou psicológica.

Deve-se, então, estabelecer estratégias para incentivar o envolvimento social de idosos e suas redes de suporte social, de modo que estes se sintam cuidados e valorizados, para que enfrentem melhor suas limitações e diminuiam a probabilidade de terem perdas em sua capacidade funcional (SANTOS et al., 2012).

Desse modo, o presente estudo se mostra altamente relevante ao se propor a pensar na importância que o envolvimento social de idosos tem para uma velhice mais saudável e com maior qualidade de vida. Outro ponto importante a ressaltar é que os relacionamentos sociais devem ser incentivados durante toda a vida e em diferentes faixas etárias, pois eles tendem a diminuir com o passar dos anos, e pessoas com um maior leque de rede social se sentem menos psicologicamente afetados quando perdem um ente querido próximo.

Estudos relacionando envolvimento social com capacidade funcional ainda são incipientes, por isso, constata-se que novos estudos dessa temática são necessários, tal como novos estudos relacionando capacidade funcional, envolvimento social e atividades avançadas da vida diária. Uma limitação do presente estudo foi o fato de ele abordar somente a literatura nacional, no entanto, ao se optar por essa variável ficou clara a necessidade de se estudar mais este tema, uma vez que só foram encontrados quatro artigos nas bases de dados pesquisadas, outros artigos se assemelhavam um pouco com a temática, mas focalizavam somente o apoio social recebido pelos idosos e não o seu envolvimento social em atividades que possam lhe gerar prazer e contribuir para a sua saúde física e psicológica e, consequentemente, em longo prazo, para a manutenção de sua capacidade funcional.

\section{Considerações finais}

As relações sociais dos idosos são determinantes para que sua capacidade funcional seja mantida por mais tempo e com maior qualidade. Com isso, são fundamentais novas investigações sobre o envolvimento social de idosos, para que se entenda melhor as relações entre capacidade funcional e envolvimento social de idosos.

A partir do presente estudo foi possível concluir que a independência funcional é um fator preditivo para o envelhecimento bem sucedido e que as relações sociais devem ser desenvolvidas ao longo da vida, para que, na velhice, elas se mantenham, uma vez que a vida social ativa do idoso em atividades prazerosas interfere positivamente na manutenção de sua capacidade funcional. 


\section{Functional capacity and social involvement in elderly: there is relationship?}

Abstract

One of today's biggest challenges is to manage the aging process and thus, the resulting increase of chronic degenerative diseases. The development of disability and dependency can be associated with the accumulation of these diseases and because the unprepared health system that may lead these seniors to the restriction of domicile and social isolation, triggering a functional decline. The aim of this study was to review the literature that regard the theme: the social involvement of seniors and functional capacity. For this, was made a literature review in the following databases: LILACS, Redalyc, BVS and SciELO. We found four articles that met the inclusion criteria, ie, regardeg the social involvement of the elderly relating it to their functional capacity. The results indicated that the social involvement of the elderly and the presence of a spouse are protective factors. That is, help to maintain the functional capacity of the elderly. From the present study it was concluded that the functional independence is a predictive factor for successful aging. Thus, it is understood that social relations should be developed throughout life, so that in old age, they remain, once an active social life can influence positively the functional capacity of these elderly. The work activities and leisure should also be valued.

Keywords: Social Interaction. Interpersonal relationship. Elderly. Functional capacity. Aging. Quality of life.

\section{Referências}

ALVES, L. C; LEITE, I. C; MACHADO, C. J. Fatores associados à incapacidade funcional dos idosos no Brasil: análise multinível. Revista de Saúde Pública, São Paulo, v. 44, n. 3, p. 468-478, maio 2010.

BRASIL. Estatuto do idoso: Lei Federal no 10.741, de $1^{\circ}$ de outubro de 2003. Brasília, DF: Secretaria Especial dos Direitos Humanos, 2004.

BRITO, T. R. P; PAVARINI, S. C. I. A relação entre o apoio social e capacidade funcional em idosos com alterações cognitivas. Revista Latino Americana de Enfermagem, Ribeirão Preto, v. 20, n. 4, p. 677-684, jul./ago. 2012.

CUPERTINO, A. P. F. B.; ROSA, F. H. M.; RIBEIRO, P. C. C. Definição de envelhecimento saudável na perspectiva de indivíduos idosos. Psicologia Reflexão e Crítica, Porto Alegre, v. 20, n. 1, p. 81-86, jan. 2007.

D'ORSI, E.; XAVIER, A. J.; RAMOS, L. R. Trabalho, suporte social e lazer protegem idosos da perda funcional: Estudo Epidoso. Revista de Saúde Pública, Florianópolis, v. 45, n. 4, p. 685-692, ago. 2011.

FARIAS, N.; BUCHALLA, C. M. A Classificação Internacional de Funcionalidade, Incapacidade e Saúde da Organização Mundial da Saúde: conceitos, usos e perspectivas. Revista Brasileira de Epidemiologia, São Paulo, v. 8, n. 2, p. 187-93, jan./fev. 2005.

FERREIRA, H. G.; BARHAM, E. J. O envolvimento de idosos em atividades prazerosas: revisão da literatura sobre instrumentos de aferição. Revista Brasileira de Geriatria e Gerontologia, Rio de Janeiro, v. 14, n. 3, p. 579-590, jul./ago. 2011.

FIDLER, M. M.; PERES, K. G. Capacidade funcional e fatores associados em idosos do sul do Brasil: um estudo de base populacional. Caderno de Saúde Pública, São Paulo, v. 24, n. 2, p. 409-415, jan. 2008. 
FREITAS, R. S. et al. Capacidade funcional e fatores associados em idosos: estudo populacional. Acta Paulista de Enfermagem, São Paulo, v. 25, n. 6, p. 933-939, jul. 2012.

GRATÃO, A. C. M. et al. Dependência funcional de idosos e a sobrecarga do cuidador. Revista da Escola de Enfermagem, São Paulo, v. 47, n. 1, p. 137-144, fev. 2013.

IBGE - INSTITUTO BRASILEIRO DE GEOGRAFIA E ESTATÍTICA. Sinopse do resultado do censo de 2013. Disponível em: <http://www.ibge.gov.br/home/estatistica/ populacao>. Acesso em: 20 jan. 2015.

NERI, A. L.; VIEIRA, L. A. M. Envolvimento social e suporte social percebido na velhice. Revista Brasileira de Geriatria e Gerontologia, Rio de Janeiro, v. 16, n. 3, p. 419-432, set. 2013.

NOGUEIRA, S. L. et al. Fatores determinantes da capacidade funcional em idosos longevos. Revista Brasileira de Fisioterapia, São Carlos, v. 14, n. 4, p. 322-329, jul./ago. 2010.

PINTO, F. N. F. R. Habilidades sociais e de enfrentamento de estresse em cuidadoras que assistem idosas acamadas, com ou sem demência. 2012. 75f. Dissertação (Mestrado em Psicologia) - Programa de Pós-Graduação em Psicologia, Universidade Federal de São Carlos, São Carlos, 2012.

PINTO, F. N. F. R.; BARHAM, E. J. Habilidades sociais e estratégias de enfrentamento de estresse: relação com indicadores de bem-estar psicológico em cuidadores de idosos de alta dependência. Revista Brasileira de Geriatria e Gerontologia, Rio de Janeiro, v. 15 , n. 3 , p. 525-539, set. 2014. Disponível em: <http://dx.doi.org/10.1590/18099823.2014.13043>. Acesso em: 20 jan. 2015.

PINTO, F. N. F. R.; BARHAM, E. J.; ALBUQUERQUE, P. P. Idosos vítimas de violência: fatores sócio demográficos e subsídios para futuras intervenções. Revista Estudos e Pesquisa em Psicologia, Rio de Janeiro, v. 13, n. 3, p. 1159-1181, dez. 2013.
PINTO, J. M.; NERI, A. L. Doenças crônicas, capacidade funcional, envolvimento social e satisfação em idosos comunitários: Estudo Fibra. Ciência \& Saúde Coletiva, Rio de Janeiro, v. 18, n. 12, p. 3449-3460, dez. 2013.

RAMOS, L. et al. Two-year follow-up study of elderly residents in S. Paulo, Brazil: methodology and preliminary results. Revista de Saúde Pública, São Paulo, v. 32, n. 5, 1998.

ROSA, F. H. M.; CUPERTINO, A. P. F. B.; NERI, A. L. Significados de velhice saudável e avaliações subjetivas de saúde e suporte social entre idosos recrutados na comunidade. Geriatria \& Gerontologia, Rio de Janeiro, v. 3, n. 2, p. 62-69, jun. 2009.

ROSA, T. E. C. et al. Fatores determinantes da capacidade funcional entre idosos. Revista de Saúde Pública, São Paulo, v. 37, n. 1, p. 40-48, fev. 2003.

SANTOS, N. M. F. et al. Qualidade de vida e capacidade funcional de idosos com osteoporose. Revista Mineira de Enfermagem, Belo Horizonte, v. 16, n. 3, p. 30-38, jul./set. 2012.

SERBIM, A. K. ; GONÇALVES, A. V. F.; PASKULIN, L. M. G. Caracterização sociodemográfica, de saúde e apoio social de idosos usuários de um serviço de emergência. Revista Gaúcha de Enfermagem, Porto Alegre, v. 34, n. 1, p. 55-63, mar. 2013.

SIMÕES, J. A. R. Bioethical reflections about the situation of elderly and their families. Acta Médica Portuguesa, Aveiro, v. 23, n. 3, p. 483-492, dez. 2010.

TEIXEIRA, I. N. D. O.; NERI, A. L. Envelhecimento bem-sucedido: uma meta no curso da vida. Psicologia - USP, São Paulo, v. 19, n. 1, p. 81-94, mar. 2008.

TORRES, L. L. Relações sociais e desempenho funcional de idosos da região metropolitana de Belo Horizonte. 2013. 67f. Dissertação (Mestrado em Saúde Pública) - Curso de Pós-Graduação em Saúde Pública, Universidade Federal de Minas Gerais, Belo Horizonte, 2013. 
TÚBERO, G. S. et al. Perfil do idoso institucionalizado. In: COELHO, F. G. M. Exercício físico no envelhecimento saudável e patológico: da teoria à prática. Curitiba: Curitiba, 2013. p. 403-410.

UESUGUI, H. M.; FAGUNDES, D. S.; PINHO, D. L. M. Perfil e grau de dependência de idosos e sobrecarga de seus cuidadores. Acta Paulista de Enfermagem, São Paulo, v. 24, n. 5, p. 685-698, 2011. 